\title{
A NOVEL REVERSIBLE WATERMARKING BASED ON AN INTEGER TRANSFORM
}

\author{
Shaowei Weng ${ }^{1}$, Yao Zhao ${ }^{1}$, Jeng-Shyang Pan $^{2}$ and Rongrong $\mathrm{Ni}^{1}$ \\ ${ }^{1}$ Institute of Information Science, Beijing Jiaotong University, Beijing 100444, P.R.China \\ ${ }^{2}$ Department of Electronic Engineering, Kaohsiung University of Applied Sciences, Taiwan
}

\begin{abstract}
A novel reversible data hiding scheme based on an integer transform is presented in this paper. The invertible integer transform exploits the correlations among four pixels in a quad. Data embedding is carried out by expanding the differences between one pixel and each of its three neighboring pixels. However, the high hiding capacity can not be achieved only by difference expansion, so the companding technique is introduced into the embedding process so as to further increase hiding capacity. A series of experiments are conducted to verify the feasibility and effectiveness of the proposed approach.
\end{abstract}

Index Terms - Reversible watermarking, Integer transform, Companding technique

\section{INTRODUCTION}

For some critical applications such as the fields of the law enforcement, medical and military image systems, it is crucial to restore the original image without any distortions after the watermark is extracted. The watermarking techniques satisfying these requirements are referred to as reversible watermarking.

The concept of reversible watermarking firstly appeared in the patent owned by Eastman Kodak [1]. After that, several reversible watermarking schemes were proposed in papers [2-8]. Xuan et al. [6] embedded data into the highfrequency wavelet coefficients by the companding technique [9] and used a preprocessing step to prevent the pixel overflow and underflow. Tian [7] expanded the value differences between two neighboring pixels to embed a bit without causing overflows or underflows. In Alatter's method [8], the differences between every two neighboring pixels in a quad were calculated according to a predefined order. Three bits were embedded into a quad by expanding the differences.

In the actual embedding processes in papers $[7,8]$, the differences less than a preset threshold were just expanded.

This work was supported in part by National Natural Science Foundation of China (No. 90604032, No. 60373028), Specialized Research Fund for the Doctoral Program of Higher Education, Program for New Century Excellent Talents in University, Specialized Research Foundation of BJTU, 973 program (No. 2006CB303104), Natural Science Foundation of Beijing (No. 4073038).
Therefore, if a small integer value was selected as the threshold, the additional information, i.e., the location map recording the expanded positions, was hardly compressed into a short bitstream due to the nearly equal possibilities for expanded and non-expanded pixels, and accordingly, it almost consumed all the available hiding capacity. In general, the hiding capacity was very low at small thresholds.

To solve this problem, a novel reversible watermarking scheme combining an integer transform with the companding technique is proposed. The transform is proposed to calculate the difference between one pixel and each of its three neighboring pixels in a quad. The companding technique is introduced so that the differences larger than or equal to the threshold can also be expanded. Accordingly, the location map can be compressed into a very short bitstream to largely increase the embedding capacity.

The rest of the paper is organized as follows. In Section 2, the integer transform is proposed. A novel reversible watermarking scheme based on the transform is presented in Section 3. The performance analysis is discussed in Section 4. The experimental results are shown in Section 5 and finally we conclude the paper in Section 6.

\section{AN INTEGER TRANSFORM}

In a grayscale image $I$, every $2 \times 2$ adjacent pixels are grouped into a quad denoted by $q=\left[\begin{array}{ll}u_{0} & u_{1} \\ u_{2} & u_{3}\end{array}\right], u_{0}, u_{1}, u_{2}, u_{3} \in \mathbb{N}$.

The forward integer transform, $T(\cdot)$, is defined as

$$
\begin{aligned}
& v_{0}=\left\lfloor\frac{u_{0}+u_{1}+u_{2}+u_{3}}{4}\right\rfloor \\
& v_{1}=u_{0}-u_{1} \\
& v_{2}=u_{0}-u_{2} \\
& v_{3}=u_{0}-u_{3}
\end{aligned}
$$

The inverse integer transform $\left[\begin{array}{ll}u_{0} & u_{1} \\ u_{2} & u_{3}\end{array}\right]=T^{-1}\left(\left[\begin{array}{ll}v_{0} & v_{1} \\ v_{2} & v_{3}\end{array}\right]\right)$ is given by:

$$
\begin{aligned}
& u_{0}=v_{0}+\left\lceil\frac{v_{1}+v_{2}+v_{3}}{4}\right\rceil \\
& u_{1}=u_{0}-v_{1} \\
& u_{2}=u_{0}-v_{2} \\
& u_{3}=u_{0}-v_{3}
\end{aligned}
$$




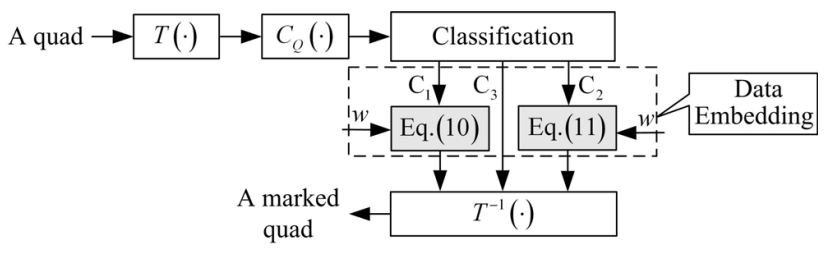

Fig. 1. Embedding process

In the following, the proof for the transform is given.

Proof: Substitute $v_{0}, v_{1}, v_{2}$ and $v_{3}$ from Eq. (1) into $u_{0}$ of Eq. (2), that is

$$
\begin{aligned}
u_{0} & =v_{0}+\left\lceil\frac{v_{1}+v_{2}+v_{3}}{4}\right\rceil \\
& =\left\lfloor\frac{u_{0}+u_{1}+u_{2}+u_{3}}{4}\right\rfloor+\left\lceil\frac{3 u_{0}-u_{1}-u_{2}-u_{3}}{4}\right\rceil \\
& =\left\lfloor\frac{u_{0}+u_{1}+u_{2}+u_{3}}{4}\right\rfloor+\left\lceil u_{0}-\frac{u_{0}+u_{1}+u_{2}+u_{3}}{4}\right\rceil
\end{aligned}
$$

Let $u_{0}+u_{1}+u_{2}+u_{3}=4 k+i$, where $0 \leq i \leq 3$ and $i, k \in \mathbb{Z}$, then we have

$$
\begin{aligned}
u_{0} & =\left\lfloor\frac{4 k+i}{4}\right\rfloor+\left\lceil u_{0}-\frac{4 k+i}{4}\right\rceil \\
& =\left\lfloor k+\frac{i}{4}\right\rfloor+\left\lceil u_{0}-k-\frac{i}{4}\right\rceil \\
& =k+u_{0}-k \\
& =u_{0}
\end{aligned}
$$

The reversibility concerning $u_{1}, u_{2}$, and $u_{3}$ can be proved by simple mathematical manipulation of $v_{1}, v_{2}$, and $v_{3}$ in Eq. (2).

\section{REVERSIBLE WATERMARKING BASED ON THE INTEGER TRANSFORM}

In this section, a reversible watermarking scheme based on the transform is presented.

\subsection{Embedding Process}

The block diagram for the proposed reversible embedding scheme is illustrated in Fig. 1. For an 8-bit grayscale image, every $2 \times 2$ adjacent pixels are grouped into a quad in raster scan order. Note that all quads do not overlap each other. $T(\cdot)$ is applied to each quad to get its transformed quad. After that, the embedding process is performed.

This process is composed of three parts: 1) Companding: a quantized compression function, $C_{Q}$, is respectively applied to $v_{1}, v_{2}$ and $v_{3} ; 2$ ) Classification: each quad is classified into one of three categories with the help of three outputs $v_{Q 1}, v_{Q 2}$ and $v_{Q 3}$;3) Data embedding: it is implemented based on the quad's category. Here, three parts are respectively explained as follows.

\subsubsection{Companding technique}

Companding technique contains a compression function $C$ and an expansion function $E$. For a signal $x, C$ and $E$ have relationship as follows:

$$
E(C(x))=x
$$

In the digital case, $C_{Q}$ and $E_{Q}$ respectively represent the quantized versions of $C$ and $E$, where $Q$ denotes quantization function. The function $C_{Q}$ adopted in this paper is given below.

$$
\begin{aligned}
x_{Q} & =C_{Q}(x) \\
& = \begin{cases}x & |x|<T_{h} \\
\operatorname{sign}(x) \times\left(\left\lfloor\frac{|x|-T_{h}}{2}\right\rfloor+T_{h}\right) & |x| \geq T_{h}\end{cases}
\end{aligned}
$$

where $\operatorname{sign}(\cdot)$ is the sign function, $T_{h}$ is a preset threshold. In this paper, $T_{h}$ is the same for all quads in an image. The expansion function is defined as

$$
E_{Q}(x)= \begin{cases}x & |x|<T_{h} \\ \operatorname{sign}(x) \times\left(2|x|-T_{h}\right) & |x| \geq T_{h}\end{cases}
$$

The companding error is calculated as follows.

$$
r=|x|-\left|E_{Q}\left(C_{Q}(x)\right)\right|
$$

For $|x|<T_{h}$, the companding error $r$ is zero. While for $|x| \geq T_{h}, r$ is non-zero, and accordingly, it needs to be collected as a part of embedded data so as to losslessly restore the image.

\subsubsection{Classification}

Each quad $q$ is classified into one of three categories: Class 1 $\left(\mathbf{C}_{1}\right)$, Class $2\left(\mathbf{C}_{2}\right)$, Class $3\left(\mathbf{C}_{3}\right)$.

$\mathbf{C}_{1}$ : For $b_{1}, b_{2}$ and $b_{3} \in\{0,1\}$, if the transformed quad $\left[\begin{array}{ll}v_{0} & \tilde{v}_{1} \\ \tilde{v}_{2} & \tilde{v}_{3}\end{array}\right]$ based on Eq. (10) satisfies Eq. (9), $q$ is classified into $\mathbf{C}_{\mathbf{1}}$.

$$
\begin{aligned}
& 0 \leq \tilde{u}_{0} \leq 255 \\
& 0 \leq \tilde{u}_{0}-\tilde{v}_{1} \leq 255 \\
& 0 \leq \tilde{u}_{0}-\tilde{v}_{2} \leq 255 \\
& 0 \leq \tilde{u}_{0}-\tilde{v}_{3} \leq 255
\end{aligned}
$$

where

$$
\begin{aligned}
& \tilde{u}_{0}=v_{0}+\left\lceil\frac{\tilde{v}_{1}+\tilde{v}_{2}+\tilde{v}_{3}}{4}\right\rceil \\
& \tilde{v}_{1}=2 v_{Q 1}+b_{1} \\
& \tilde{v}_{2}=2 v_{Q 2}+b_{2} \\
& \tilde{v}_{3}=2 v_{Q 3}+b_{3}
\end{aligned}
$$

$\mathbf{C}_{\mathbf{2}}$ : For $q \notin \mathbf{C}_{\mathbf{1}}$, if $\left[\begin{array}{cc}v_{0} & \tilde{v}_{1} \\ \tilde{v}_{2} & \tilde{v}_{3}\end{array}\right]$ given by Eq. (11) satisfies Eq. (9), $q$ is considered to be in $\mathbf{C}_{\mathbf{2}}$.

$$
\begin{aligned}
& \tilde{v}_{1}=2\left\lfloor\frac{v_{1}}{2}\right\rfloor+b_{1} \\
& \tilde{v}_{2}=2\left\lfloor\frac{v_{2}}{2}\right\rfloor+b_{2} \\
& \tilde{v}_{3}=2\left\lfloor\frac{v_{3}}{2}\right\rfloor+b_{3}
\end{aligned}
$$


$\mathbf{C}_{3}$ : The rest quads belong to $\mathbf{C}_{\mathbf{3}}$.

Note that in the classification process, values 0 and 1 need to be assigned to $b_{i}, 1 \leq i \leq 3$, respectively.

For each $q$ in $\mathbf{C}_{1}$, if whichever of $v_{1}, v_{2}$ and $v_{3}$ is larger than or equal to $T_{h}$, it is classified in set $\mathbf{C}_{\geq T_{h}}$. Otherwise, it is considered to be in set $\mathbf{C}_{<T_{h}}$.

\subsubsection{Data Embedding}

The embedding process consists of two steps:

\section{Step 1: Constructing to-be-embedded bitstream}

A location map is created by assigning ' 1 ' to all the quads in $\mathbf{C}_{1}$ and ' 0 ' to the others. And then it is losslessly compressed by an arithmetic encoder to form a bitstream $\mathcal{L}$ with an unique EOS symbol at the end. Let $L_{S}$ be the bit length of $\mathcal{L}$.

For each $v$ in $\mathbf{C}_{\geq T_{h}}$, its companding error $r$ given by Eq. (8) is collected into another bitstream $\mathcal{R}$. Thereby, there are four parts of information to be embedded: 1) bitstream $\mathcal{L}$; 2) bitstream $\mathcal{C}$ containing the LSBs of $v_{1}, v_{2}$ and $v_{3}$ of each quad in $\mathbf{C}_{\mathbf{2}}$;3) bitstream $\mathcal{R} ; 4$ ) the payload. These four parts are combined together into a bitstream $\mathcal{W}$.

\section{Step 2: Embedding the bitstream}

For each $q$ in $\mathbf{C}_{\mathbf{1}}$, bits in $\mathcal{W}$ are embedded into $v_{1}, v_{2}$ and $v_{3}$ by left shifting and replacing the LSBs as described in Eq. (10). For each $q$ in $\mathbf{C}_{2}$, bits in $\mathcal{W}$ are used to replace the LSBs of $v_{1}, v_{2}$ and $v_{3}$ as in Eq. (11). All the quads in $\mathbf{C}_{\mathbf{3}}$ are kept unaltered. After all the bits in $\mathcal{W}$ are embedded, $T^{-1}(\cdot)$ is applied to get the marked image $I_{w}$.

\subsection{Extraction Process}

Every $2 \times 2$ adjacent pixels in the marked image $I_{w}$ are grouped into a quad in the same way as in embedding. $T(\cdot)$ is applied to each quad to get its transformed one $q^{\prime}=\left[\begin{array}{cc}v_{0} & \tilde{v}_{1} \\ \tilde{v}_{2} & \tilde{v}_{3}\end{array}\right]$.

The extraction process consists of two steps:

\section{Step 1: Location map extraction}

For each $q^{\prime}$, with $\tilde{v}_{1}, \tilde{v}_{2}$ and $\tilde{v}_{3}$ as inputs of Eq. (11), if the outputs satisfy Eq. (9), this quad is classified into set $\overline{\mathbf{C}}_{\mathbf{3}}$. Otherwise, it belongs to set $\mathbf{C}_{\mathbf{3}}$.

The LSBs of $\tilde{v}_{1}, \tilde{v}_{2}$ and $\tilde{v}_{3}$ in $\overline{\mathbf{C}}_{3}$ are collected into a bitstream $\mathcal{W}$. By identifying the EOS symbol in $\mathcal{W}$, the bits from the start until EOS are decompressed by an arithmetic decoder to retrieve the location map. For $q^{\prime}$ in $\overline{\mathbf{C}}_{3}$, if its location map value is $1, q^{\prime}$ is classified into the set $\tilde{\mathbf{C}}_{\mathbf{1}}$. Otherwise, it belongs to set $\tilde{\mathbf{C}}_{\mathbf{2}}$. The bits after EOS in $\mathcal{W}$ are identified as bitstream $\mathcal{R}$, bitstream $\mathcal{C}$ and the payload.

\section{Step 2: Restoration}

For each $q^{\prime}$ in $\tilde{\mathbf{C}}_{\mathbf{1}}$, its original difference value $v_{1}$ is restored according to Eq. (12).

$$
v_{1}= \begin{cases}v_{Q 1} & \left|v_{Q 1}\right|<T_{h} \\ \operatorname{sign}\left(v_{Q 1}\right) \times\left(2\left|v_{Q 1}\right|-T_{h}+w\right) & \left|v_{Q 1}\right| \geq T_{h}\end{cases}
$$

where $v_{Q 1}=\left\lfloor\frac{\tilde{v}_{1}}{2}\right\rfloor, w \in \mathcal{R}$.

For $q^{\prime} \notin \tilde{\mathbf{C}}_{\mathbf{1}}$, its $v_{1}$ is retrieved as follows:

$$
v_{1}= \begin{cases}2 \times\left\lfloor\frac{\tilde{v}_{1}}{2}\right\rfloor+w & \tilde{v}_{1} \in \tilde{\mathbf{C}}_{\mathbf{2}} \\ \tilde{v}_{1} & \tilde{v}_{1} \in \mathbf{C}_{\mathbf{3}}\end{cases}
$$

where $w \in \mathcal{C}$.

The restoration of $v_{2}, v_{3}$ is similar to that of $v_{1}$. Finally, $T^{-1}(\cdot)$ is applied to reconstruct the original image $I$.

\section{PERFORMANCE ANALYSIS}

The difference modification caused by the companding technique and the data-hiding capacity are analyzed in this section.

\subsection{Difference Modification}

For each $v$ in $\mathbf{C}_{\geq T_{h}}$, its marked value $v_{w}$ is equal to $\left(2 \times v_{Q}+\right.$ $w), w \in\{0,1\}$. Let $d$ be the difference between $v_{w}$ and $v$.

If $v \geq T_{h}$, then we have the following equations

$$
\begin{gathered}
r=v-\left(2 \times v_{Q}-T_{h}\right) \\
d=v_{w}-v=\left(T_{h}+w-r\right)
\end{gathered}
$$

If $v \leq-T_{h}$, then

$$
\begin{gathered}
r=-v-\left(-2 \times v_{Q}-T_{h}\right) \\
d=v_{w}-v=\left(r+w-T_{h}\right)
\end{gathered}
$$

To conclude from above, when $v \in \mathbf{C}_{\geq T_{h}}$, each difference $d$ is in the range of $\left[-T_{h}, T_{h}+1\right]$. While for $v \in \mathbf{C}_{<T_{h}}$, $d$ is in the interval $\left(-T_{h}, T_{h}+1\right)$. In conclusion, each $d$ would not exceed the range $\left[-T_{h}, T_{h}+1\right]$.

\subsection{Hiding Capacity}

The maximum hiding capacity is given by:

$$
\begin{aligned}
D & =3\left\|\mathbf{C}_{\mathbf{1}}\right\|-L_{S}-\|R\| \\
& =\left\|\mathbf{C}_{\geq T_{h}}\right\|+\left\|\mathbf{C}_{<T_{h}}\right\|-L_{S}-\|R\| \\
& =\left\|\mathbf{C}_{<T_{h}}\right\|-L_{S}
\end{aligned}
$$

Where the operator $\|\cdot\|$ represents the length of a sequence or the cardinality of a set.

For each $v$ in $\mathbf{C}_{\geq T_{h}}$, it can carry one bit, but meanwhile, it will introduce 1-bit companding error need to be embedded, so $\left\|\mathbf{C}_{\geq T_{h}}\right\|=\|R\|$. Thereby, it has no direct contribution to increase the hiding capacity. However, they are useful to enhance $\left\|\mathbf{C}_{\mathbf{1}}\right\|$, and accordingly, the location map can be efficiently compressed into a short bitstream to get a small $L_{S}$. Finally, the data hiding is largely increased. 


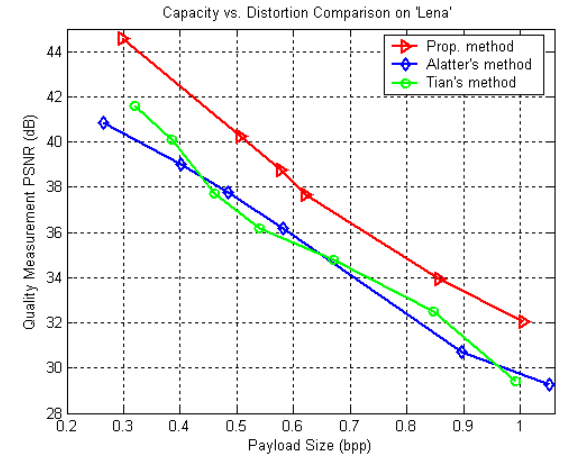

Fig. 2. Capacity vs. distortion comparison on 'Lena'

\section{EXPERIMENTAL RESULTS}

For comparison, we experimented our method, Tian's method [7], and Alatter's method [8] on several commonly used images including 'Lena' and 'Baboon' with size $512 \times 512$. Our method and Alatter's can achieve embedding rates of close to $0.75 \mathrm{bpp}$ without multiple embedding. Multiple embedding was done while testing Tian's so as to achieve embedding rates above $0.5 \mathrm{bpp}$.

The capacity vs. distortion comparisons among our method, Alatter's and Tian's are shown in Fig. 2 and Fig. 3.

As reported in Fig. 2, the top curve is the proposed method. By embedding a payload of the same size (bit length), the PSNR value of our method is about 1-3dB greater than that of the others. For example, the high embedding capacity of close to $0.3 \mathrm{bpp}$ (bit per pixel) is achieved with above $44 \mathrm{~dB}$ in PSNR. However, at the same embedding rate, the PSNR values of the other two methods are both less than $42 \mathrm{~dB}$.

'Baboon' represents images with large areas of complex texture, so the experimental results are not good in Alattar's method and Tian's method. However, due to no pixels at both ends of its histogram image, most pixels can be still available for embedding at small $T_{h}$. For instance, in our method, the PSNR value above $44 \mathrm{~dB}$ can still be obtained with the embedding rate over $0.1 \mathrm{bpp}$. Our method still outperforms Alattar's and Tian's at almost all PSNRs.

\section{CONCLUSIONS}

A novel reversible data hiding scheme based on an integer transform is presented in this paper. The companding technique is introduced so as to largely increase the number of quads in $\mathbf{C}_{\mathbf{1}}$, and accordingly, the data-hiding capacity is increased especially at small thresholds. From the experimental results, our performance is superior to the others'.

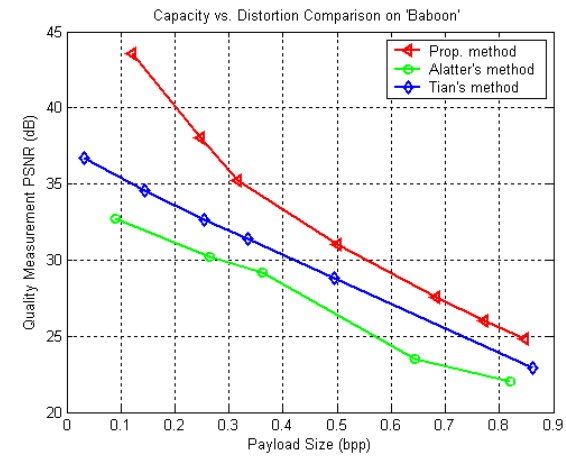

Fig. 3. Capacity vs. distortion comparison on 'Baboon'

\section{REFERENCES}

[1] C.W. Honsinger, P. Jones, M. Rabbani, and J. C. Stoffel, Lossless Recovery of an Original Image Containing Embedded Data, US patent:6278791, 2001.

[2] M. Goljan, J. Fridrich, and R. Du, "Distortion-free data embedding for images," in 4th Information Hiding Workshop, LNCS, New York, 2001, vol. 2137, pp. 27-41, Springer-Verlag.

[3] M.U. Celik, G. Sharma, A.M. Tekalp, and E. Saber, "Reversible data hiding," in Proceedings of IEEE International Conference on Image Processing, 2002, vol. 2, pp. $157-160$.

[4] M.U. Celik, G. Sharma, and A.M. Tekalp, "Lossless watermarking for image authentication a new framework and an implementation," IEEE Transaction on Image Processing, vol. 15, pp. 1042-1049, 2006.

[5] M. Thodi and J.J. Rodrłguez, "Prediction-error based reversible watermarking," in Proceedings of IEEE International Conference on Image Processing, 2004, vol. 3, pp. 1549-1552.

[6] G.R. Xuan, C.Y. Yang, Y.Z. Zhen, and Y.Q. Shi, "Reversible data hiding using integer wavelet transform and companding technique," in Proceedings of International Workshop on Digital Watermarking, 2004.

[7] J. Tian, "Reversible data embedding using a difference expansion," IEEE Transaction on Circuits and Systems for Video Technology, vol. 13, pp. 890-896, 2003.

[8] A.M. Alattar, "Reversible watermark using difference expansion of quads," in Proceedings of IEEE Internation Conference on Acoustics, Speech, and Signal Processing, 2004, vol. 3, pp. 377-380.

[9] M. Veen, F. Bruekers, A. Leest, and S. Cavi, "High capacity reversible watermarking for audio," in Proceedings of the SPIE, 2003, vol. 5020, pp. 1-11. 